\title{
Neonatal brain damage and intelligence in rats'
}

\author{
KEITH J. HAYES ${ }^{2}$ \\ LOMA LINDA UNIVERSITY, LOS ANGELES COUNTY GENERAL HOSPITAL
}

\begin{abstract}
Rats were reared in a complex environment containing a varicty of doors and passageways. Periodic observation showed that brain damage reduced spontaneous exploitation of the chiromment; and subsequent testing showed that the resulting deficit in experience was reflected in inability to negotiate doors and passageways to reach a goal.
\end{abstract}

This exploratory investigation was based on an interpretation of intelligence (Hayes, 1962) which is unusual in two respects. First, the general nature of the Binet-type test was taken as an operational definition of intelligence. Such a test does not require new learning or problem solving, but simply requires the $S$ to employ the learned skills and information which he has accumulated prior to the time of testing. The second departure from tradition is the assumption that most of the learned skills and information which constitute intelligence are acquired in the course of activity which is intrinsically motivated, and independent of such drives as hunger and fear. It was hypothesized that this experience-producing activity depends on built-in neural structures, and that early damage to these structures will reduce the amount of subsequent experience-producing activity, thus retarding intellectual development.

\section{Method and Results}

Rats were reared for their first 45 days in an environment which provided various opportunities for intrinsically motivated learning. Exploitation of these opportunities was evaluated by frequent observation; and the amount actually learned was finally measured with Binet-type test items.

One litter of rats was used, including four normals and five with electrolytic lesions made in the anterior part of the brain on the day of birth. The rat's brain is in a relatively early stage of development at birth: Undifferentiated neuroblasts are present, mitosis is common, and many neurons have short, unbranched processes. Lesions in such an embryonic brain may be subject to some repair; however, they may also induce abnormality in remote parts of the brain. Thus it was found, when the brains were sectioned, that simple surgery had produced complex brain damage. The rat with the most extensive damage had a primary lesion centered in the septum, with considerable loss in the caudate, cortex, hippocampus, thalamus, several major fiber tracts, and the amygdala. The corpus callosum was small and displaced to a position entirely posterior to its normal location. The other damaged brains had similar but less extensive abnormalities.

The experimental environment was complex; but complexity alone would not serve the present purpose, which required environmental features with three specific characteristics. (1) They must elicit a response from a normal rat; (2) they must permit the rat to learn something as a result of this response; and (3) they must be adaptable to use in a test situation, so the occurrence of learning can be objectively determined. With these requirements in mind, nine devices which could function as doors or passages were built into the environment. In practice, four of them elicited very little response, and only the five satisfactory items will be discussed. (A naive control group tested on these items at 46 days of age performed very poorly, showing that they did, in fact, require experience.)

One of the items was a compact, five-choice-point, multiple-T, alley maze. All of the rats were seen in this apparatus frequently during the 45 days of free access to it. At 46 days they were confined in a separate cage, without water, for $16 \mathrm{~h}$, then returned to the environment, one at a time, at a point from which the standard water-bottle location could be reached only by passing through the maze. All but one of the rats got through in less than $1 / 2 \mathrm{~min}$, usually without errors. The exceptional rat-in this and all other tests-was the one with the greatest brain damage. He took $8 \mathrm{~min}$ to get through, and made eight errors in the process.

The barricade test consisted of a doorway blocked by four light pieces of wood, two on each side. All but one rat had often been seen moving the wooden barriers about, and chewing on them. When tested, using the same procedure as with the maze, they moved the barriers aside and got through the door within $1 \mathrm{~min}$. The rat with the largest lesion was never seen in contact with this item until the test session, when he took $10 \mathrm{~min}$ to get through.

The tunnel-digging test required the rat to dig his way down to a doorway buried in sawdust. All but one of the rats had often been seen in this apparatus, and several had been seendigging their way through it. When tested, they got through within $1 \mathrm{~min}$. The rat with the largest lesion had occasionally been seen in the apparatus, but had never been seen digging. When tested, he failed to get through in the $10 \mathrm{~min}$ allowed.

The revolving-door test involved a modified activity wheel. It had an entrance hole in one wall and an exit hole, displaced $180^{\circ}$, in the other wall, so a rat could enter the device from the right, rotate it one half turn, and leave at the left. All but one of the rats had been seen in this apparatus very frequently, and several 
of them had been seen passing through it in the intended fashion. When tested, they got through within $1 \mathrm{~min}$, and only one of them turned the wheel a single superfluous revolution in the process. The severely-braindamaged rat was seen in this apparatus only once, on the day before the test session. When tested, it took him $10 \mathrm{~min}$ to get through, and he turned the wheel 84 excess revolutions.

The paper-door test was a passageway blocked by a replaceable sheet of heavy wrapping paper which could be torn away by the rats. On the day before testing, the plain paper was replaced with a sheet perforated by a small hole in the center. Some of the rats contacted the paper occasionally, but they never tore it until the last day, after the perforation was introduced. Then, all but one of them began tearing, and they used up 20 sheets by the end of the day. Several rats were seen tearing the paper away and then going through the door. When tested, most of them got through within $2 \mathrm{~min}$, and all within 7 min. The severely-brain-damaged rat was never seen near this door before the test session, and failed to get through in $15 \mathrm{~min}$.

When the Binet-type testing was completed, three forms of "spontaneous locomotor activity" were measured. The activity wheel showed no clear effect of brain damage; however, locomotion in the open-field test and the stabilimeter cage were affected drastically and in opposite directions. The most severely braindamaged rat displayed about $5 \%$ as much exploratory activity as the least-active normal rat during two 10-min sessions in the open field. However, during nine days in the stabilimeter cage, his activity was 10 times normal. One other rat, with slightly less extensive brain damage, was also far below normal in exploratory behavior, very hyperactive in the stabilimeter cage, and consistently below average on all Binet-type items.

Some of the brain-damaged rats performed normally on all Binet-type test items. However, it cannot be concluded that their intellectual development was unimpaired, since the tests were probably too easy to detect moderate deficits at 46 days. The early spontaneous behavior of the normal rats suggested that they might have passed the tests much sooner. (A fully developed Binet-type test would, of course, include items of graded difficulty presented at various ages.) Discussion

This preliminary investigation demonstrates the feasibility of a new approach to the experimental study of intelligence. One brain-damaged rat displayed a behavioral syndrome corresponding closely to the motivational-experiential conception of intellectual retardation. He was hyperactive, in a stereotyped way, but his interaction with his environment was limited to walking through some parts of it. He was never seen pushing, pulling, digging, scratching, or tearing anything, or chewing anything except food. He falled to learn things that normal rats learned, and performed very pooriy on a test of Binet-type intelligence.

\section{Reference}

HAYES, K. J. Genes, drives, and intellect. Psychol Rep., 1962, 10, 299-342.

Notes

1. Supported by NIH grant HD00919.

2. Present address: White Memorial Medical Center, Los Angeles, Calif., 90033. 